\title{
Study on Mandarin plosives Airflow and Air-pressure of Junior high school Based on PAS6600
}

\author{
Xiaohui Yang ${ }^{a}$, Yonghong Li ${ }^{\text {b, * }}$ and Dawa Pengcuo ${ }^{c}$ \\ Key Lab of China's National Linguistic Information Technology, Northwest University for Nationalities, \\ Lanzhou, 730030 China \\ a547686498@qq.com, blyhweiwei@126.com, cdwpc@163.com \\ *corresponding author
}

Keywords: Junior, plosives, Mean Expiratory Airflow, Mean Peak Air Pressure, Expiratory Volume.

\begin{abstract}
In this paper we explore the difference between male and female in middle school , making use of the PAS6600 and the experimental data with the microcosmic angle concludes that: 1) In junior high school students, mandarin Chinese EAD of aspirated sound is greater than unaspirated sound. 2) The MEA of male is greater than the MEA of female, the MEA of aspirated sound is greater than the MEA of unaspirated sound. 3) The EV of aspirated sound is greater than unaspirated sound. The EV of male is always greater than the female students, which has a lot to do with men's higher lung capacity. 4) The Male's MPAP is greater than the female's MPAP and the MPAP of aspirated sound is less than the MPAP of the unaspirated sound.
\end{abstract}

\section{Introduction}

The theoretical research on Mandarin plosives is very deep and extensive in our country, and there are many researches on Mandarin plosives in the direction of experimental phonetics. Wu Zongji did unaspirated/ aspirated features experiment of standard Chinese consonants in the 1980s' This article reveals the mandarin differences between aspirated and unaspirated ${ }^{[1]}$. In 2008, Hu Axu did the consonants and vowels based on airflow /air pressure signal found the correspondence between physiological and acoustic parameters ${ }^{[2]}$. In 2010 Lv Shiliang used phonatory aerodynamic system discussed the plosive and affricate of standard Chinese consonants ${ }^{[3]}$, In 2013, Liu Yali made a dynamic analysis of the characteristics of the vowel characteristics of Chinese common speech in middle school students, indicating that the gender and age have significant influence ${ }^{[4]}$ In this paper, the contents of the study are Simple vowels, and the subjects are junior high school students, using Pronator Aerodynamic System6600 explore the aerodynamic characteristics of mandarin, try to find out the difference between airflow and air pressure during ages.

\section{Experimental Description}

The experiment is mainly based on the mandarin (b, p, d, t, g, k), To explore the variation of aerodynamic parameters in the pronunciation of junior high school students, The subjects selected a male(15 years old) and a female(13 years old) with a standard mandarin, required to have a normal voice and no hearing impairment. The experimental site was carried out in the speech lab of northwestern national university, Using the PAS6600 equipment produced by American KAY company for signal collection, asking the pronunciations to understand the collection principle and the pronunciation list in advance for better completion of the experiment, changing the position of the pressure tube in the mouth according to the different changes in the pronunciation, and each word pronounced three times according to the word list. 


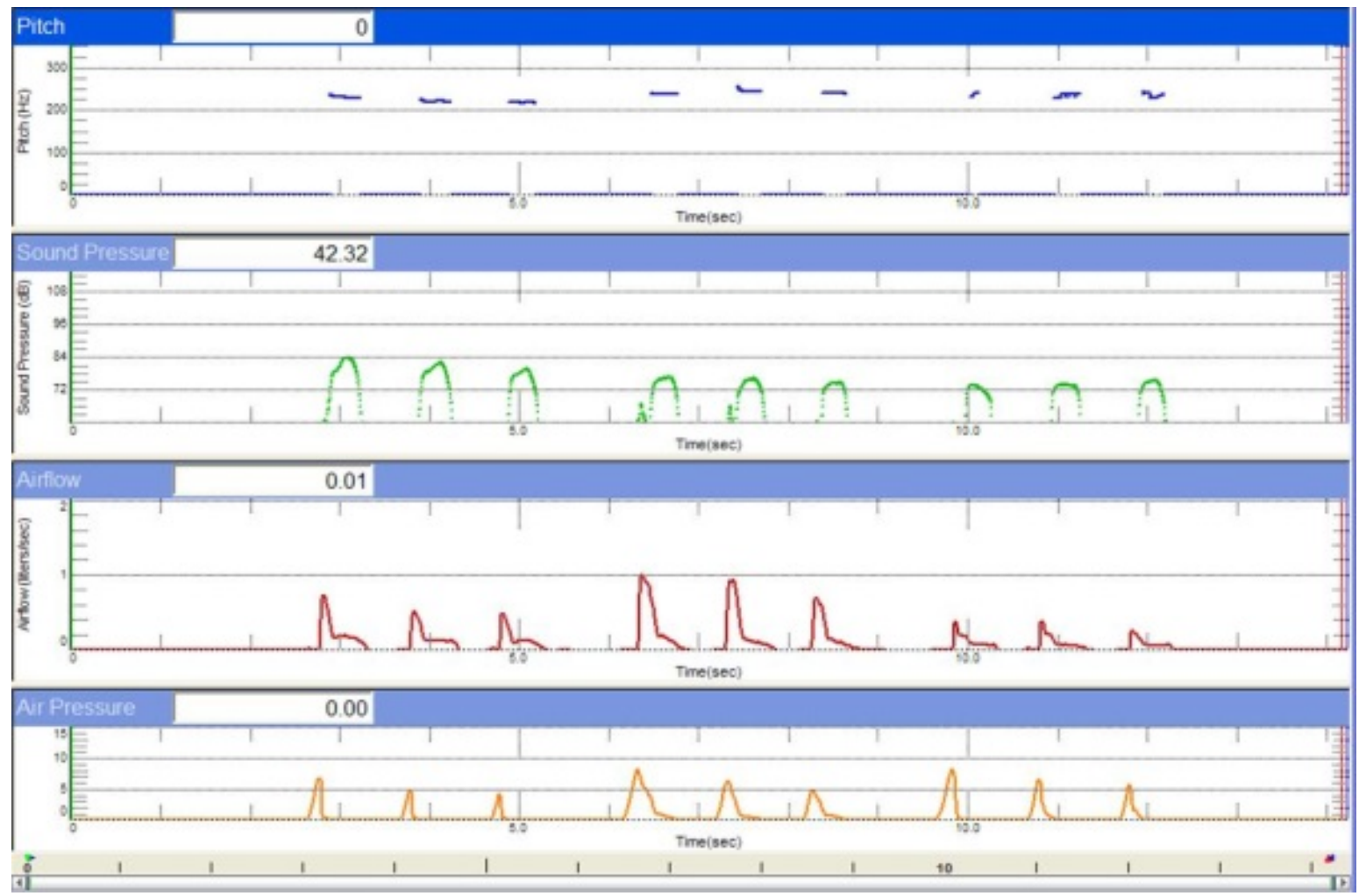

Figure 1: The 'p' of Mandarin plosives for junior high school students

\section{Experimental Analysis}

The aerodynamic parameters of the junior high school students can be obtained through data extraction of the intact experiment, as shown in table 1, different parameters represent different physiological characteristics. EAD(sec): Expiratory Airflow Duration represents the beginning of a syllable consonant to the end of a vowel. MPAP $\left(\mathrm{cm} / \mathrm{H}_{2} \mathrm{O}\right)$ : Mean Peak Air Pressure means the most atmospheric pressure is the average of the atmospheric pressure in the oral cavity. MEA(Lit/Sec): Mean Expiratory Airflow represents the average speed of exhalation during pronunciation. EV(Liters): Expiratory Volume represents the total amount of air flow in the pronunciation process , usually proportional to the duration of the pronunciation.

Table 1: Mandarin plosives of Junior high school Parameter table

\begin{tabular}{|c|c|c|c|c|c|c|c|c|c|c|c|c|}
\hline \multirow{2}{*}{$\begin{array}{l}\text { Vowel } \\
\text { parameters }\end{array}$} & \multicolumn{2}{|c|}{ b } & \multicolumn{2}{|r|}{ p } & \multicolumn{2}{|r|}{ d } & \multicolumn{2}{|r|}{$\mathrm{t}$} & \multicolumn{2}{|r|}{ g } & \multicolumn{2}{|c|}{$\mathrm{k}$} \\
\hline & male & female & male & female & male & female & male & female & male & female & male & female \\
\hline EAD & 0.19 & 0.22 & 0.24 & & 0.28 & 015 & 0.45 & 025 & 0.14 & 0.04 & 0.24 & 0.17 \\
\hline MPAP & 7.02 & 6.94 & 6.09 & & 7.54 & 7.0 & 6.17 & 1 & 8.86 & 7.40 & 7.59 & 7.25 \\
\hline MEA & 0.28 & 0.06 & 0.44 & 0.17 & 0.18 & 0.08 & 0.33 & 0.22 & 0.26 & 0.11 & 0.50 & 0.33 \\
\hline EV & 0.05 & 0.01 & 0.09 & 0.05 & 0.05 & 0.02 & 0.10 & 0.08 & 0.07 & 0.04 & 0.12 & 0.06 \\
\hline
\end{tabular}

\subsection{Expiratory Airflow Duration and Mean Expiratory Airflow}

Junior high school students are in a special transition phase, most students vocal cords start to change at this age, the vocal organs grow rapidly and the vocal cords are swelling. There are subtle variations in the female vocal organs, but no significant differences. The flow of air produced in the process of human pronunciation is provided by the lungs and air passes through the throat and causes the vocal cords to vibrate, the breathing apparatus can interfere with airflow, the internal organs of the vocal organs are rubbed, they eventually flow out through the mouth. 

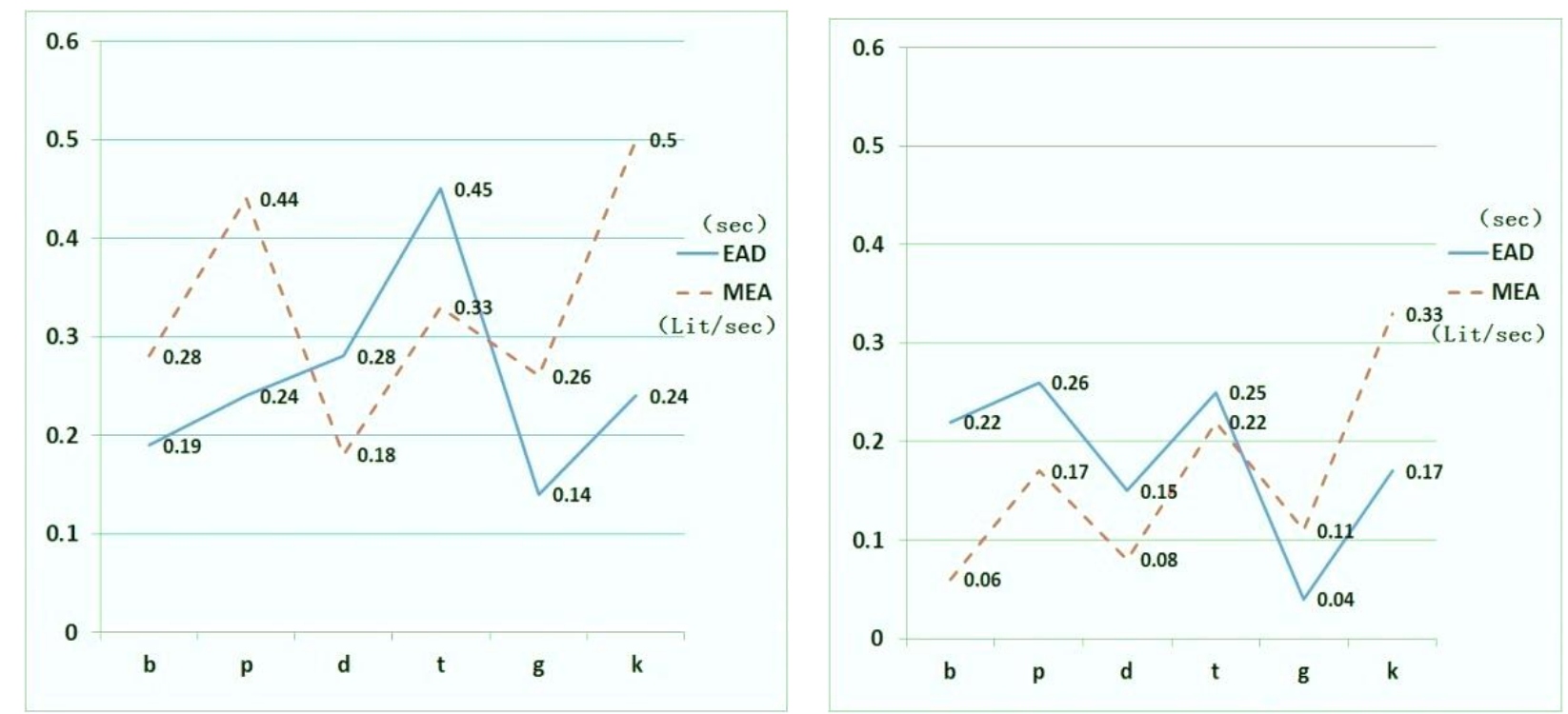

Figure 2: The EAD and MEA of male students Figure 3: The EAD and MEA of female students

The airflow inside the cavity of Mandarin plosives(b, p, d, t, g, k, )is blocked experiences three process: Beginning to hinder, Continuing to hinder, Removing obstacles. The resistance phase flow is completely blocked and cannot be passed, until the removing obstacles phase, the air flow bursts out. The place of articulation of mandarin plosives $b$ and $p$ is mouth, the place of articulation of $d$ and $\mathrm{t}$ is tongue tip, the $\mathrm{g}$ and $\mathrm{k}$ is root of tongue, the difference between them is the airflow ${ }^{[5]}$.

EAD represents the duration of the exhalation, According to the average parameter value of the male and female of table 1 we can see that the EAD of $p>$ EAD of $b$, the EAD of $t>$ EAD of $d$, the EAD of $\mathrm{k}>\mathrm{EAD}$ of $\mathrm{g}$, so we can draw a conclusion that in Mandarin plosives of Junior high school, the EAD of aspirated sound > unaspirated sound, This is obvious that the time of exhaling air flow is significantly longer than the unaspirated sound during the pronunciation process. For the same pronunciation methods of different gender comparison can be found that Female subjects of the EAD $b$ and $p>$ male subjects of the EAD $b$ and $p$, male subjects of the EAD $d$ and $t>$ Female subjects of the EAD $d$ and $t$, male subjects of the EAD $g$ and $k>$ Female subjects of the EAD $g$ and $\mathrm{k}$.

MEA represents the average rate of exhalation air flow during pronunciation, As shown in figure 2 and figure3 we can see clearly that : In the six plosives of mandarin Chinese, the MEA of male $>$ the MEA of female, this suggests that men are more likely to exhale higher airflow rate when they are in the process of phonation, which is linked to the male physiological mechanism. The average parameters of the male and female MEA was evaluated we can discover that: the MEA of $p>$ the MEA of $b$, the MEA of $t>$ the MEA of $d$, the MEA of $k>$ the MEA of $g$, this shows that in the pronunciation Mandarin plosives of Junior high school the MEA of aspirated sound $>$ the MEA of unaspirated sound.

\subsection{Mean Peak Air Pressure and Expiratory Volume}

The pressure value is used to measure the pressure of the water column in the mouth, generally speaking, the pressure value of $3 \mathrm{cmH}_{2} \mathrm{O}$ can vibrate the vocal cords, In the normal course of speaking the air pressure in the mouth is usually above $5 \mathrm{cmH}_{2} \mathrm{O}$. The MPAP in this paper is used to measure the air pressure of middle - middle school students, the difference of plosives is that the blocking phase is completely blocked, the air pressure value of the mouth is at its peak, In the resistance phase pressure value drops out immediately. 

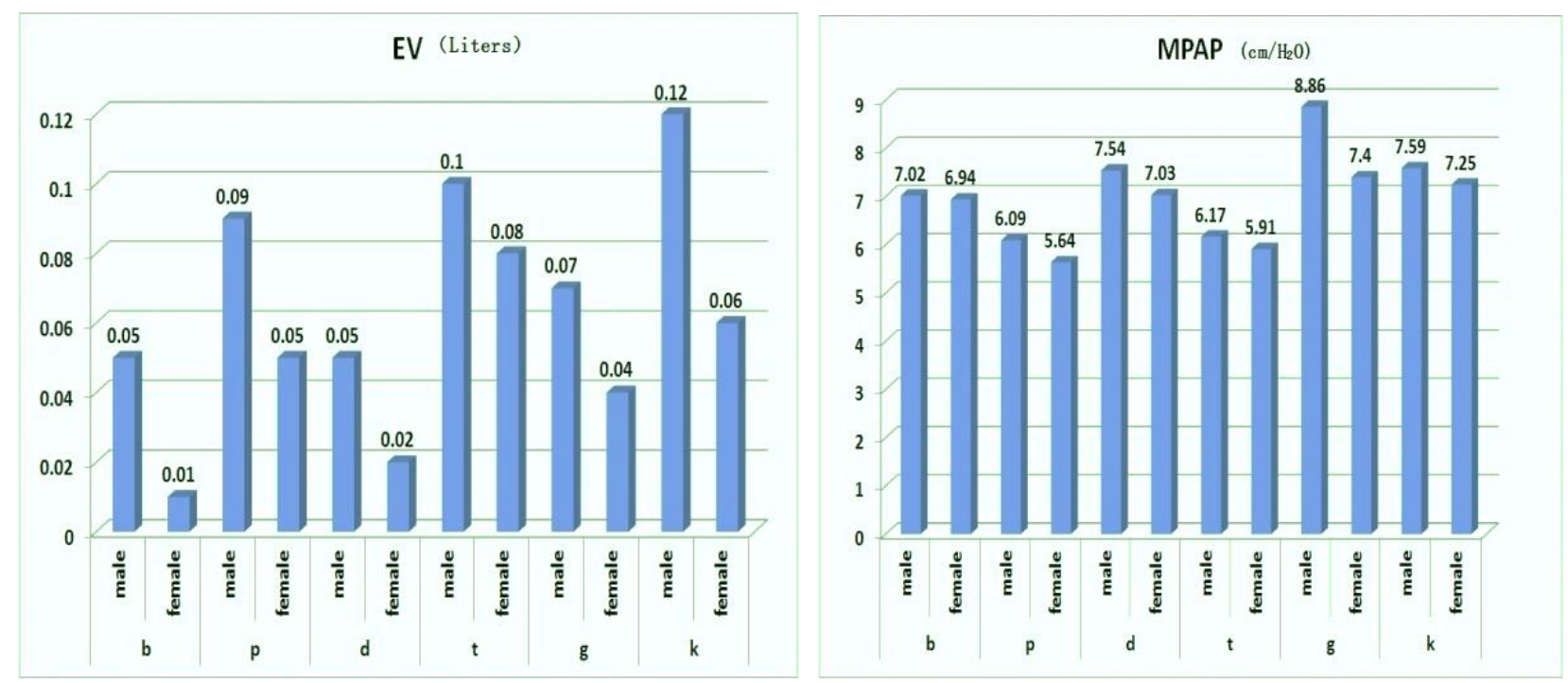

Fig4: The EV of Mandarin plosives for junior Fig 5: The MPAP of Mandarin plosives for junior

Figure 4 reflects that the change of the total volume of the air flow between different genders of Chinese mandarin in junior high school students is not only concerned with the differences in the pronunciations, but also the gender differences. Exhaled airflow volume represents the total airflow rate in the process of pronunciation, and the pronunciation time will be has an impact on the exhaled airflow volume. According to the figure 4 we can see that the total exhaled airflow of Plosive b, p, d, t and g, $\mathrm{k}$ is not same, the same pronunciation for men and women are not same too, But after the parameter values for the average of men and women $p$ is greater than $b$, $t$ greater than $\mathrm{d}$ and $\mathrm{k}$ is greater than the $\mathrm{g}$, this indicates that the total volume air flow of aspirated sound is greater than unaspirated sound. From the picture, we can also see that: the total amount exhalation air flow of male is always greater than the female students, which has a lot to do with men's higher lung capacity.

Figure 5 shows the change of MPAP between different sexes in junior high school students. can be seen that the average pressure peak value of men and women is above $5 \mathrm{cmH}_{2} \mathrm{O}$, and regardless of the expiration men's MPAP is greater than women on MPAP, It is indicated that the pressure peak in the elimination stage of middle school male students in the peak, the MPAP of $b>$ the MPAP of $p$, the MPAP of $d>$ the MPAP of $t$, the MPAP of $g>$ the MPAP of $k$, Therefore, we can conclude that the MPAP of aspirated sound is less than the MPAP of the unaspirated sound.

\section{Conclusion}

In this paper the aerodynamic parameters of Chinese mandarin for junior high school students are analyzed by experimental data compared between different genders and different pronunciations. The experiment draws a conclusion that in junior high school students, mandarin Chinese EAD of aspirated sound is greater than unaspirated sound. The MEA of male is greater than the MEA of female, the MEA of aspirated sound is greater than the MEA of unaspirated sound. The EV of aspirated sound is greater than unaspirated sound. The EV of male is always greater than the female students, which has a lot to do with men's higher lung capacity. The Male's MPAP is greater than the female's MPAP, the MPAP of aspirated sound is less than the MPAP of the unaspirated sound.

\section{Acknowledgments}

This article is subsidized by Fok Ying Tung Education Foundation (Grant No. 151110)

\section{References}

[1] Wu Zong Ji, Talk about "difference characteristics" of the pronunciation of mandarin Chinese and their mutual relations, "Chinese language" in 1980 
[2] $\mathrm{Hu}$ a $\mathrm{Xu}$, The study of the mandarin consonants and vowels based on airflow /air pressure signal, [D], 2010.4

[3] Lv Shi liang, Research on mandarin plosive and affricate sound air pressure [D], 2012.4

[4] Liu Ya Li, A dynamic analysis of the characteristics of the vowel characteristics of Chinese common speech in middle school students [J], 2013.8

[5] Lin Tao, Wang li jia. Phonetics profile [M]. Beijing: higher education press, 2014.7 